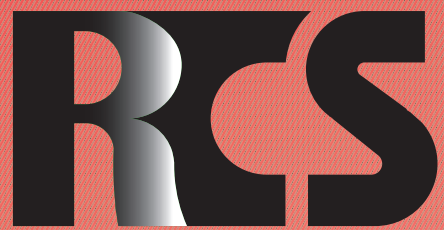

Depósito legal ppi $201502 Z U 4662$

Esta publicación científica en formato digital es continuidad de la revista impresa Depósito Legal: pp $197402 Z U 789$

- ISSN: 1315-9518 • ISSN-E: 2477-9431

Revista de Ciencias Sociales

Universidad del Zulia. Revista de la Facultad de Ciencias Económicas y Sociales Vol. XXVII. No. 2

Abril-Junio 2021

Esta publicación científica en formato digital es continuidad de la revista impresa Depósito Legal: pp $197402 Z$ Z789 ISSN: 1315-9518 

J. (2021). Compromiso organizacional y factores demográficos que propician el ausentismo laboral en franquicias de Barranquilla-Colombia. Revista de Ciencias Sociales (Ve), XXVII(2), 163-179.

\title{
Compromiso organizacional y factores demográficos que propician el ausentismo laboral en franquicias de Barranquilla- Colombia
}

\author{
Mora, Yeimer* \\ Romero, Carmen** \\ Muñoz, Ignacio*** \\ Sierra, Joaquín****
}

\section{Resumen}

El desafío actual del liderazgo y la gestión organizacional es crear herramientas útiles a través de las cuales los empleados puedan determinar los objetivos organizacionales e integrarlos en los proyectos empresariales. La presente investigación estuvo dirigida a analizar los elementos del Compromiso organizacional y su relación con los factores demográficos que propician el ausentismo laboral del sector franquicias de comida rápida en Barranquilla, Colombia. La metodología fue de tipo descriptiva, de campo, la muestra seleccionada fue de 155 sujetos, quienes laboran en restaurantes de comida rápida del distrito Barranquilla. A dicha población se le aplicó un cuestionario integrado por 54 ítems, con escala Likert. Los resultados muestran que los elementos del compromiso hacia la organización están muy presentes en el proceso de integración laboral, tales como identificación organizacional, involucramiento en el trabajo y la lealtad hacia la organización, ello indica que existe un gran sentido de pertenencia. Aunado a ello se concluye que, aunque existe un nivel alto de ausentismo laboral, este se relaciona con factores sociodemográficos, tales como el hecho de que el personal femenino tiene compromiso con sus hijos, apoyo a la familia, el estado civil y la edad. En algunas ocasiones la distancia del lugar de trabajo.

Palabras clave: Ausentismo laboral; compromiso; factores socio-demográficos; involucramiento; sentido de pertenencia.

\footnotetext{
Cursante del Doctorado en Contabilidad. Docente de la Universidad de la Costa, Colombia. E-mail: ymora@cuc.edu. co iD ORCID: https://orcid.org/0000-0002-7903-2202

** Doctora en Ciencias Políticas. Doctora en Ciencias de la Educación. Docente del Tecnológico de Antioquia, Colombia. E-mail: carmenhelena2007@gmail.com iD ORCID: https://orcid.org/0000-0002-1768-2947

*** Doctor en Historia. Docente de la Universidad Andrés Bello, Chile. E-mail: Ignacio.munoz@unab.cl (iD) ORCID: https://orcid.org/0000-0002-3758-5861

**** Magister en Tributación. Docente de la Universidad Libre - seccional Cartagena, Colombia. E-mail: pablosierra07@ gmail.com iD ORCID: https://orcid.org/0000-0002-9601-9085
}

Recibido: 2020-12-07 · Aceptado: 2021-02-24 


\title{
Organizational commitment and demographic factors that encourage absenteeism in franchises in Barranquilla- Colombia
}

\begin{abstract}
The current challenge of organizational leadership and management is to create useful tools through which employees can determine organizational objectives and integrate them into business projects. This research was aimed at analyzing the elements of Organizational Commitment and its relationship with the demographic factors that promote absenteeism in the fast food franchise sector in Barranquilla. The methodology was descriptive, field, the selected sample was 155 subjects, who work in fast food restaurants in the Barranquilla district. A questionnaire made up of 54 items, with a Likert scale, was applied to this population. The results show that the elements of commitment to the organization are very present in the labor integration process, such as organizational identification, involvement in work and loyalty to the organization, which indicates that there is a great sense of belonging. In addition to this, it is concluded that, although there is a high level of absenteeism from work, this is related to sociodemographic factors, such as the fact that female personnel are committed to their children, support for the family, marital status and age. Sometimes the distance from the workplace.
\end{abstract}

Keywords: Labor absenteeism; commitment; socio-demographic factors; involvement; sense of belonging.

\section{Introducción}

El desafío actual del liderazgo y la gestión organizacional, es crear herramientas útiles a través de las cuales los empleados puedan determinar los objetivos organizacionales e integrarlos en los proyectos de la empresa. Desde esta perspectiva, el desarrollo de estrategias exitosas de recursos humanos conduce a una adecuada gestión de las mismas, para obtener ventajas sostenibles y duraderas en el tiempo.

De acuerdo, a lo planteado, se evidencia que tanto las organizaciones, como las personas, tienen objetivos por alcanzar. Las empresas están orientadas a cumplir la misión para lo cual fueron creadas, coordinada dicha misión con metas, objetivos y valores. En opinión de Parra, et al. (2019) alcanzar el propósito, y dependerá de sus integrantes, por lo cual, si el empleado está de acuerdo con el líder, pero además acepta sus valores, produciendo así el desempeño esperado al realizar la tarea, este provoca un intercambio de esfuerzos y resultados. Se ha observado, que el compromiso organizacional de los empleados con la organización, se relaciona directamente con las acciones que se perciban desde la empresa y de sus integrantes.

Dentro de esta perspectiva, Parra, et al (2018) manifiestan que la gestión de recursos humanos tiene una gran responsabilidad en las empresas, adquiriendo la función primordial de consolidar el ambiente laboral fomentando el compromiso organizacional, ofreciendo a los empleados los elementos motivadores para que estos se sientan identificados con la actividad laboral que realizan y de acuerdo al estudio, disminuir el ausentismo laboral como variable interviniente en el proceso de gestión productiva. 
Según Hellriegel y Slocum (2010), el logro de las metas de la organización requiere que exista un compromiso desde el discernimiento de los empleados, en este sentido, la organización debe ser leal a los empleados, pero además debe generar contribuciones positivas de los mismos. Destacando, que la actitud hacia el trabajo es más amplia que la satisfacción, porque se aplica no solo a toda la organización, sino al desarrollo de las actividades y a los procesos personales.

En este orden de ideas, Robbins y Coulter (2014) han considerado que el compromiso organizacional, es concebido como un proceso que determina el grado donde un empleado genera identificación y relación con una organización determinada, orientándose al cumplimiento de metas. Destacan además, que como línea general estos trabajadores quieren estar asociado a esa empresa. En el mismo sentido, hay un alto involucramiento con el puesto de trabajo, en pro de identificarse con el cargo específico de cada empleado, asimismo, un alto compromiso organizacional expresa solidarizarse con la organización empleadora.

Para, Newstrom (2011), el compromiso organizacional se adquiere en el transcurso del tiempo, en la medida en que el empleado obtiene mayor información acerca de la empresa, por ende, los gerentes deben establecer las condiciones para conducir a los empleados a un alto nivel de compromiso. Bajo este contexto, se puede afirmar que existen muchos caminos para que los gerentes incrementen y mantenga el potencial de compromiso de los trabajadores, un ejemplo de ello podría ser el sistema de recompensas e incentivos que tenga una empresa, incluyendo capacitación y respeto al trabajo del individuo.

Dentro de este marco, Hellriegel y Sloucum (2010) manifiestan que el compromiso organizacional se concibe como la intensidad de la participación de un empleado, su identificación con la organización y se caracteriza por la creencia o aceptación de sus metas y valores, la disposición para realizar esfuerzos importantes en beneficio de la organización, con el consiguiente deseo de pertenecer a la misma.

García, et al. (2017a), sostienen que, como resultado, aparece el sentido de identidad de los empleados con la organización y la satisfacción con el trabajo que realizan, caracterizado por una alta subjetividad personal; por lo cual, el departamento de recursos humanos tiene el rol de empoderarlos. De acuerdo con la visión organizacional, de lograr compromiso y participación del empleado, así como la supervivencia de la organización, surge la tendencia del fenómeno denominado ausentismo laboral. Este se considera un fenómeno que se da en la conveniencia de la relación entre organizaciones laborales e individuos.

En función de lo planteado, Rodríguez (1999) manifiesta que el ausentismo laboral, implica ausencia de una persona de su puesto de trabajo, en horas que corresponden a un día laborable, dentro de la jornada legal. A este respecto, el ausentismo se relaciona con la pérdida temporal de horas o días de trabajo, independientemente de las causas que lo originen.

Asimismo, Robbins y Coulter (2014), considera al ausentismo laboral como una variable dependiente, pues es el resultado, conjuntamente con la rotación, la productividad y la satisfacción laboral, de la interacción entre diferentes factores que actúan a nivel del individuo, el grupo al que pertenece y el sistema organizativo.

En otras palabras, el absentismo es un fenómeno que expresa de forma indirecta el conflicto entre los trabajadores y su entorno laboral, que se da más o menos en todos los ámbitos económicos. El cual en opinión de García, Durán y Prieto (2017), se hace característico por los problemas que rodean la existencia del ser humano; destaca así, la presencia de un sector donde impacta la actividad empresarial; es el caso del sector servicios, representado por el de comida rápida en establecimientos denominados franquicias.

En Colombia, actualmente existe una gran variedad de organizaciones de este tipo, dado que la globalización y la dinámica empresarial, ha conllevado a los individuos a 
optar por estas alternativas de alimentación, originado por factor tiempo, imposibilidad de recorrer largas distancias, pero además precios y variedad; por ende, la expansión de tales negocios, se ha vuelto una necesidad creciente de las sociedades actuales.

Consecuentemente, y debido al tipo de atención prestada y la concepción de comida rápida, en estas empresas se hace necesaria la contratación de grandes volúmenes de empleados, lo que le permite cubrir la magnitud de sus operaciones; en ellas, la fuerza laboral es cuantiosa, la misma se integra en su mayoría por jóvenes y mujeres. Los mismos son capacitados continuamente, en pro de generar involucramiento y sentido de pertenencia, además del arraigo a la cultura organizacional, promoviendo valores a través de la prestación de servicios de calidad. Forjado como parte esencial de la identificación del empleado con la organización a la cual pertenece.

El sector franquicias de comida rápida, se caracteriza por tener estructuras bien definidas, formalizadas a través de la certificación de los procedimientos de trabajo visiblemente establecidos; dado que estas son empresas, en su mayoría internacionales, aunque en Colombia existen franquicias originales de servicios colombianos. Ellas están orientadas a la mejora continua, y procesos de integración del personal. Sin embargo, las jornadas laborales están integradas por actividades continuas y agotadoras a las que están sometidos los trabajadores.

Las características particulares de la fuerza laboral, en su mayoría son jóvenes menores de 25 años de edad, mujeres y en condición social de estrato 1 y 2 así como con distancias largas desde su vivienda. Particularidades que los hacen sujetos proclives a circundar en el fenómeno del ausentismo y de la rotación laboral, faltando al trabajo con alta frecuencia, presentando problemas en cuanto al cumplimiento de los horarios de trabajo, lo cual para los líderes, manifiesta poco compromiso con la organización, poniendo en riesgo la productividad organizacional.

Para cubrir las necesidades, se ha contratado mayor cantidad de personal operativo para satisfacer las demandas de los turnos laborales, suplir las ausencias producidas por las faltas al trabajo mencionadas y ocasionando incrementos en los costos de contratación, aumento en los costos de nómina, reducción de las ganancias, incumplimiento en las políticas de las empresas y, por ende, insatisfacción de la clientela. En virtud de lo expuesto anteriormente, surgió la necesidad de analizar los elementos del Compromiso organizacional y su relación con los factores demográficos del ausentismo laboral.

\section{Elementos del compromiso organizacional.}

El compromiso organizacional lo define Newstrom (2011), en relación al grado de identificación de un empleado con su organización, pero también, se relaciona con el deseo de seguir participando activamente en ella, e incluso lo cataloga como lealtad del empleado. En otro orden de ideas, Robbins y Coulter (2014) así como, Méndez, Morua y Hernández (2018), lo conceptualizan como un proceso psicológico donde un empleado se identifica con una organización y sus metas, y quiere seguir formando parte de ella, hasta sentirla propia, involucrándose activamente con ella y dirigiendo esfuerzos en beneficio de la organización; teniendo claro el coste que implicaría dejarla, estimulando así el deseo de permanencia dentro de la misma. Aunado a ello, Hellriegel y Slocum (2010), lo asumen como una actitud ante el trabajo, integrada por tres componentes básicos, que se observan en la Figura I: 


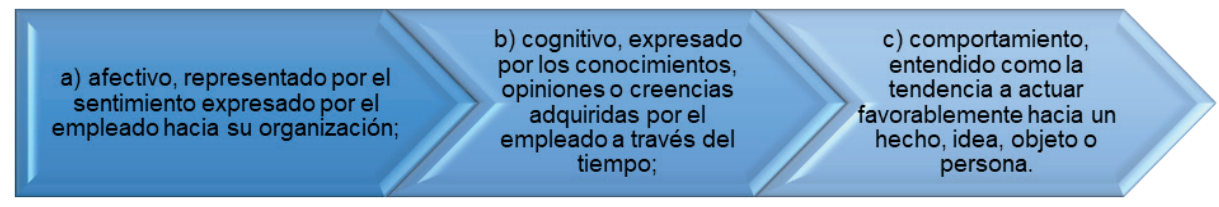

Fuente: Hellriegel y Slocum (2010).

\section{Figura I: Componentes básicos del compromiso organizacional}

Sin embargo, Schemehom, Hunt y Osborn (2008), le da a estos elementos o factores la connotación de variables intervinientes, las cuales determinan el grado de compromiso presentado por el individuo hacia la organización empleadora; pudiendo agruparse en distintas categorías; así, enumera variables inherentes a las características propias del individuo; variables relacionadas con el ambiente laboral, donde se desarrolla la persona; y variables asociadas a las características organizacionales o del grupo. En cuanto a las variables individuales, se refieren a la edad, sexo, nivel educativo, estado civil, número de hijos, antigüedad en la organización y el puesto, salario, competencias del individuo, las expectativas, los valores, entre otros.

Dentro de este marco, estos elementos del compromiso organizacional no trabajan de forma independiente o aislada, sino entrelazada, interactuando entre sí, a través de la suma de factores individuales y organizacionales; estos factores o variables intervinientes ayudan a los empleados a esmerarse en su trabajo, buscando la calidad de todo lo concerniente a su labor.

A este respecto, Robbins y Coulter (2014), puntualizan que el compromiso organizacional implica un proceso complejo, cuyo núcleo se centra en aspectos idiosincrásicos del individuo como: Su escala de valores, creencias, cultura y sentimientos, sustentado por Durán, García-Guiliany y Parra-Fernández (2018), se involucran diferentes elementos, entre ellos los del ser; concebido como un proceso iniciado cuando el individuo se hace miembro de la organización, evolucionando desde la identificación con los aspectos organizacionales y el involucramiento con la misma, hasta el deseo o sentimiento de pertenencia.

a. Identificación: Corresponde a la primera etapa del proceso de compromiso organizacional, la cual es definida por Robbins y Coulter (2014), como la implicación psicológica, emocional y conceptual de las personas que implica la aceptación de los valores y objetivos organizacionales, logrando con ello la obtención de las metas establecidas por la empresa al igual que compartir la responsabilidad de las mismas, generando mejores resultados en las labores, mediante el cambio y la adhesión a metas personales estimuladoras de un mejor desempeño. La identificación, está constituida por los siguientes aspectos, que se aprecian en la Figura II. 

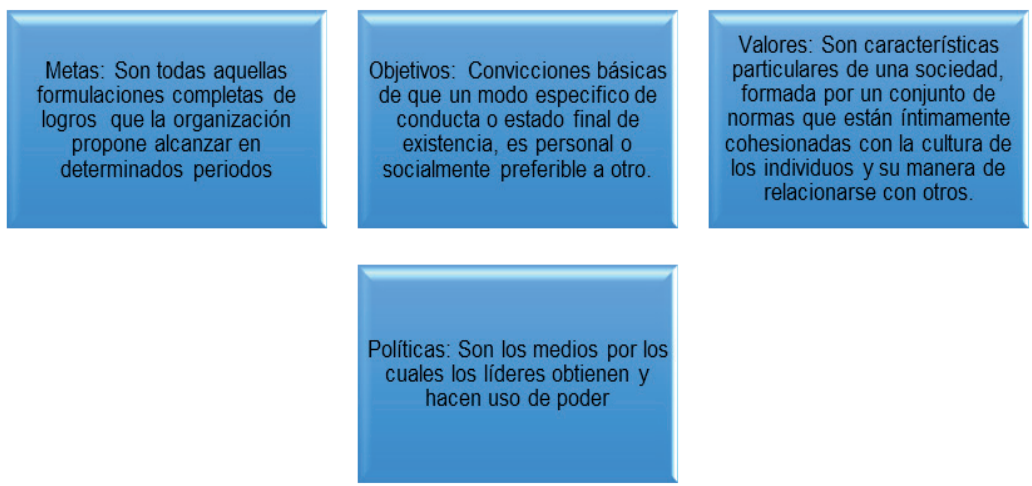

Fuente: Robbins y Coulter (2014).

\section{Figura II: Características para promover la identificación organizacional}

Es conveniente resaltar lo planeado por Prieto, et al. (2016), quienes sostienen que, a través de la identificación, los empleados pueden aprovechar al máximo su creatividad y talentos, para completar mejor el trabajo, porque no hay presión para realizarlo, sino para asumir sus responsabilidades, el propósito y los objetivos de la organización, porque se siente parte de la empresa. b. Involucramiento: Robbins y Coulter (2014), consideran que el involucramiento del empleado con su organización, constituye la segunda fase del proceso de compromiso organizacional, mediante su sentido de obligación y lealtad, traduciéndose en un mayor esfuerzo dirigido al beneficio de la organización. En este sentido, los mismos autores define los elementos que lo integran, en la Figura III.

Decisiones: Constituyen el medio para lograr algún resultado o para resolver algún problema.

Participación: característica del empleado que lo faculta a auto dirigirse y tomar decisiones de forma autónoma en beneficio de la organización: conjunto de estrategias y actividades del empleado implementadas en pro del fortalecimiento de la organización, en cuanto a producción, calidad de servicios, imagen, entre otros.
Esfuerzo: Empeño físico o mental necesarios que refieren a la responsabilidad de cumplir una labor que conlleve a la consecución de un objetivo.
Interés: Importancia, valor encaminado hacia el logro o la propensión de un fin.
Rendimiento: Producción física o

intelectual que un individuo presenta en cualquier actividad que se le asigne para el mejor funcionamiento de la organización

Fuente: Robbins y Coulter (2014).

\section{Figura III: Elementos del involucramiento}


De similar enfoque, este deseo de involucramiento se manifiesta en el impulso de la gente hacia la eficacia personal, según Newstrom (2011), en base a la certeza de poseer las capacidades necesarias para desempeñar una tarea, cumplir las expectativas puestas en determinadas funciones, realizar una contribución significativa o enfrentar exitosamente una situación difícil.

Es decir, hoy en día, muchos empleados buscan activamente oportunidades para participar en decisiones laborales importantes, con el fin de aportar sus talentos e ideas al éxito de la organización, siempre que tengan un conocimiento pleno de la visión y misión de la organización (Paz, et al. 2016). Bajo este contexto, las organizaciones deben brindar a los miembros del personal tal involucramiento, puesto que esto otorgará beneficios a los empleados y a la organización como un todo, en el sentido de que se les otorga una oportunidad para participar activamente de acuerdo a sus roles.

c. Sentido de pertenencia: Robbins y Coulter (2014), lo conciben como el proceso donde los miembros de una organización establecen conexiones con la cultura, los valores y la estructura organizacional, a través de sus actitudes, y asumen que son propios, al tiempo que establecen una interacción psicológica entre las necesidades personales y organizacionales. A su vez, esta dimensión se divide en los siguientes indicadores, visualizados en la Figura IV, que determinan la etapa final del compromiso organizacional.

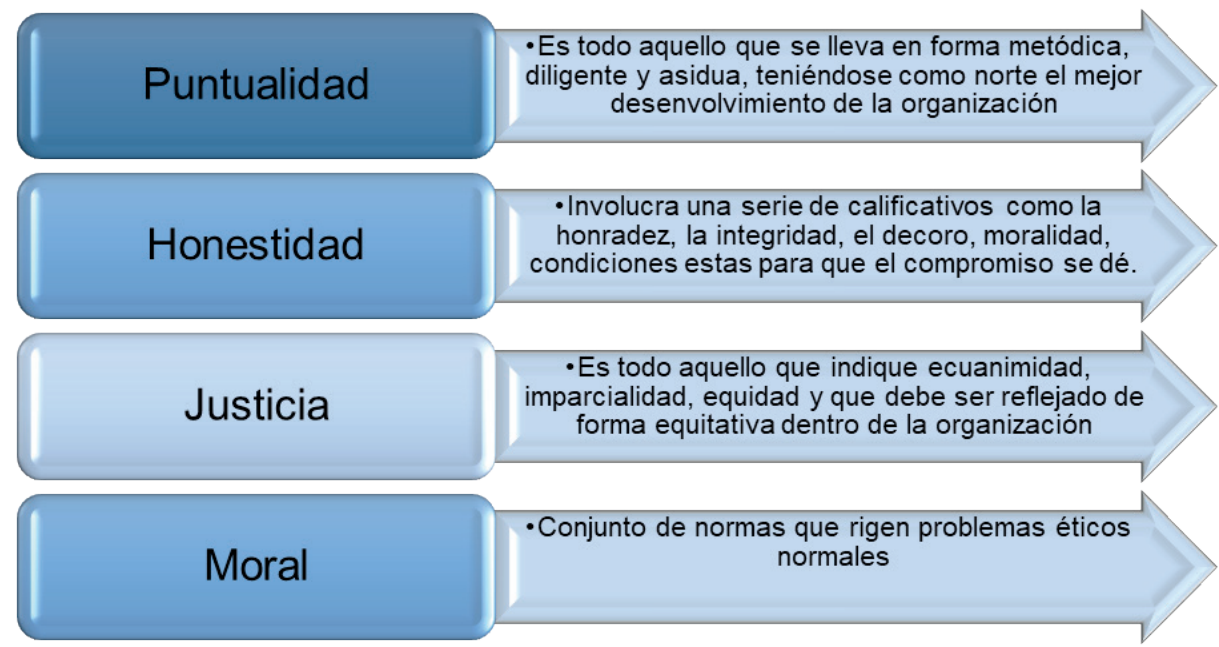

Fuente: Robbins y Coulter (2014).

Figura IV: Indicadores de sentido de pertenencia

De acuerdo a lo anterior, en la organización debe haber una alianza sólida entre el empleado y la misma, donde éste sienta que es parte de la empresa, pueda aplicar su inteligencia así como creatividad, y así desarrollarse individualmente, buscando el éxito de la organización y el propio. De esta forma, se obtendrán empleados más competitivos, colaboradores, con apego a la organización, consolidado con los objetivos, metas, visión y misión de la misma, a través de equipos auto dirigidos de trabajo (Duran, et al., 2017; Cabrera y Díaz, 2019).

Es así, como el compromiso que los 
trabajadores tienen con la organización hace que éstos alcancen el nivel de productividad más alto. Según López, García y Cardeño (2017), se consideran parte de la organización $y$, por ende, tratan de alcanzar los objetivos y metas establecidas por esta para el beneficio de la misma y de su propio beneficio. Por lo tanto, se hace necesario un clima de trabajo adecuado, agradable para todos los integrantes de la organización, donde éstos se sientan libres de expresar su talento, inteligencia, creatividad, que sean capaces de trascender barreras, con el fin de alcanzar las metas y objetivos de la organización; ser solidarios con sus compañeros, implementar mejoras continuas, lo que en opinión de MartínezVentura, et al. (2017), permita fomentar los estilos de liderazgo positivo para que exista la participación activa de los empleados dentro de la organización.

Por lo tanto, para las organizaciones, la relación entre el compromiso organizacional y la rotación es uno de los factores más importantes, porque cuanto más fuerte es el compromiso del empleado con la organización, es menos probable que se retire. Los hechos han demostrado que el alto compromiso está asociado a un bajo ausentismo. Los empleados con fuertes compromisos organizacionales, suelen tener una mayor asistencia al trabajo.

Más importante aún, las personas dedicadas tienden a estar impulsadas por objetivos y pierden menos tiempo de trabajo, lo que tiene un impacto positivo en las métricas de productividad típicas, así lo establecen Hellriegel y Slocum (2010). Es decir, que ese compromiso les permite posicionarse como personas que aumentan la rentabilidad en las organizaciones.

\section{Factores demográficos del ausentismo laboral}

El ausentismo laboral, para Amorós (2007), se centra en la definición de absentismo, porque involucra una serie de comportamientos, y no existe una conexión obvia entre estos. Hasta ahora, no existe una teoría que pueda cubrir todas las explicaciones sobre este tema. Lo común entre el ausentismo laboral y las ausencias en el trabajo, es el tiempo de trabajo perdido, aunque la ausencia al trabajo es un acto involuntario y el ausentismo laboral es voluntario. Molinera (2006), destaca que los factores que condicionan el ausentismo son los siguientes, que se observan en la Figura V.

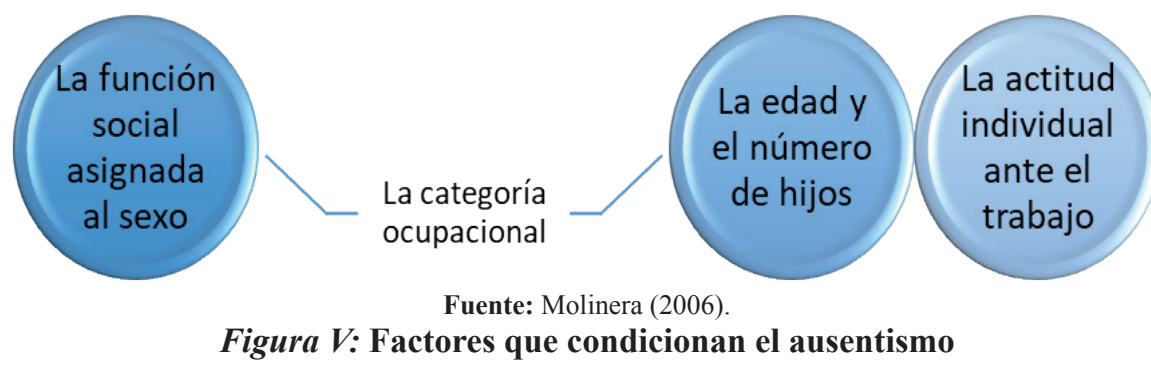


Destaca, Molinera (2006), que en muchas ocasiones las ausencias femeninas han sido mayores que la de los hombres, puesto que la mayoría de las empleadas mujeres tienen el doble de actividades, dado que disponen de su horario de trabajo, atención a procesos con sus hijos, permisos extra para ir al médico con ellos, entre otras funciones. Por otro lado, los hombres tienen funciones diferentes y en muchos casos no retrasarán el ritmo de trabajo, pero el riesgo de trabajo como es alto, podría en algunos momentos propender al ausentismo. A continuación, se vinculan los factores relacionado en esta propuesta.

a. Demográficos: Para Martínez (2016), se relacionan con los conocimientos, experiencias, habilidades, y actitudes concebidas como características que influyen en el proceso de adaptación de las personas a su ambiente, lo que permitirá enfrentarse a los estímulos que le exigen respuestas consecuentes ante tales demandas. Cada individuo percibe una estructura mentalmente, actuando de acuerdo a sus capacidades sobre las fuentes de estrés interior y exterior a él. Algunos responden satisfactoriamente ante las demandas, algunos otros no; en tanto, alguien puede percibir una situación como desafío y otro la puede sentir como una amenaza.

Entre las habilidades facilitadoras resaltadas por Duran (2017), se encuentran el sexo, edad, estado civil, la existencia de hijos, la antigüedad en el puesto y en la profesión; con todo, las variables sociodemográficas en total constituyen un porcentaje importante del ausentismo. En cuanto al sexo, indica López, et al. (2005) que se observan diferencias importantes en función de las actitudes de despersonalización, registrando los varones una puntuación más alta.

Es así que las mujeres son moldeadas socialmente para cuidar, educar y encargarse de los niños, la pareja, los ancianos, tal vez por esa razón sea menos proclive a responder en forma irritable a las personas con problemas. En cuanto a la edad, hay una clara relación entre este factor y el ausentismo; indica Peiró, et al. (2008) que es mayor cuando los trabajadores son jóvenes.
En relación al estado civil, el apoyo socio-emocional que se recibe por parte de familiares y amigos, influyen en forma significativa en disminuir las posibilidades de ausentismo, más que el estado civil. Según López, et al. (2005), la familia y los compañeros de trabajo se complementan para aliviar el estrés laboral o prevenirlo. Estos individuos, influyen para que los sujetos sean más expertos en el trato con los demás y así menos propensos al ausentismo.

Otro aspecto importante, es la existencia o no de hijos, en el cual Molinera (2006) afirma que los sujetos sin hijos puede que tengan mayor tendencia a usar el trabajo como fuente de vida social, lo que los hace implicarse excesivamente con la gente del ámbito laboral y mayor riesgo de sufrir de estrés. En cuanto a la antigüedad en el puesto y la profesión en concordancia con el factor edad, los profesionales nuevos son más jóvenes e inexpertos, por lo tanto, poseen menos estrategias de afrontamiento para prevenir las situaciones estresantes.

b. Distancia al trabajo: Bajo el criterio de Amorós (2007), en lo relativo a la distancia al trabajo, se requiere en este punto analizar lo que tenga que ver con dos cosas importantes: Primero, lo que hace la organización en materia de contratación con respecto al manejo de empleados que vivan cerca de la localidad en la que se encuentra; segundo, los problemas externos que el empleado tenga que enfrentar para llegar a su sitio de trabajo.

\section{Metodología}

El objetivo de la presente investigación fue analizar los elementos del Compromiso organizacional y su relación con los factores demográfico del ausentismo laboral. La misma es de tipo descriptiva, puesto que describe los elementos que conforman el compromiso organizacional, además de los factores demográficos que influyen en el ausentismo. En cuanto al diseño de la investigación, se ejecutó un diseño no experimental de campo, en el cual se expresa que la información o los 
datos fueron obtenidos directamente de la realidad. Además, se analizó el compromiso y el ausentismo, estableciendo sus características específicas sin participar o manejar el contexto y se ejecutó una sola medición.

Dentro del diseño no experimental se tipifica como transeccional, porque su fin fue analizar los elementos del compromiso organizacional de los trabajadores de los restaurantes de comida rápida en la ciudad de Barranquilla, Colombia, en un momento específico en el tiempo. Para efectos de esta investigación, la población estuvo constituida por 155 empleados operativos que laboran en 10 franquicias de comida rápida ubicadas en centros comerciales de la zona norte de Barranquilla, (Buena Vista 1, Buena vista 2 y Viva), para ello se ejecutó un muestreo accidental, considerado como el muestreo no probabilístico que no utiliza la aleatoriedad en una o más de sus etapas y que en él se toman las muestras disponibles a mano. En este sentido, la población esta representadas por locales con empleados de los establecimientos de las franquicias con mayor índice de atención de clientes, durante fines de semanas y días festivos, verificado durante visita exploratoria inicial.

Para recolectar la información requerida, se utilizó la técnica de observación mediante encuesta, con un instrumento conformados por 54 afirmaciones con selección de respuesta en escala tipo Likert, cuyas ponderaciones van del 1 al 5, asignándoles una escala estadística de medición ordinal. En este sentido, es importante destacar cómo un item se basa en una proposición enfocada a expresar una idea positiva o negativa respecto de un fenómeno interesado en conocerse y ante la cual se solicita la reacción del sujeto. El encuestado manifiesta un grado de frecuencia con cada item. Cada respuesta tiene una puntuación favorable o desfavorable; la puntuación total del instrumento, se determinó por la suma algebraica de las puntuaciones de las respuestas del individuo a todos los items que se le han presentado.

Asimismo, el instrumento fue validado a través del análisis discriminante de items, aplicando una prueba piloto a treinta y dos (32) sujetos con características similares a la población seleccionada, con el fin de descartar aquellos items que no fueran válidos para el estudio. Al respecto se utilizó la siguiente fórmula:

t calculada $=\frac{\text { Media del Grupo Alto }- \text { Media del Grupo Bajo }}{\sqrt{\frac{\text { Varianza Grupo Alto }}{n-1}+\frac{\text { Varianza del Grupo Bajo }}{n-1}}}$

Durante el procedimiento, fueron comparadas la $\mathrm{T}$ calculada $(\mathrm{Tc})$ por cada item con la $\mathrm{T}$ teórica $(\mathrm{Tt})$ o $\mathrm{T}$ de la tabla; si el valor de Tc es mayor que el de la de $\mathrm{Tt}$, el item se mantiene en el cuestionario, si por el contrario el valor de Tc es menor, se eliminara, es decir: Si Tc > Tt: el item se acepta para la escala final. $\mathrm{Si} \mathrm{Tc}<\mathrm{Tt}$, el item se elimina de la escala. Asimismo, es necesario señalar que la $\mathrm{T}$ teórica es proporcionada por la tabla $\mathrm{T}$ de Student, la cual se obtuvo interceptando el error seleccionado con los grados de libertad.

De esta manera, fueron comparadas la $\mathrm{T}$ calculada con la $\mathrm{T}$ teórica establecida de acuerdo con el nivel de error o significancia de 0,05 y $(8+8)-2=14$ grados de libertad. El resultado de la prueba determinó que el instrumento fue válido al aceptar aquellos items donde la $\mathrm{T}$ calculada fue mayor que la $\mathrm{T}$ teórica $(\mathrm{Tc}>\mathrm{Tt})$, en este caso no se eliminó ningún item, por lo tanto, el cuestionario se mantuvo con todos sus reactivos.

Con el fin de calcular la confiabilidad de los cuestionarios diseñados, se seleccionó el coeficiente de Alfa Cronbach, el cual se aplica en una sola administración del instrumento de medición, representado por valores entre 0 y 1 . Se evaluó la posición de treinta y dos sujetos (32) que poseían características similares a las de la muestra definitiva, los valores obtenidos establecen unos intervalos que oscilan entre 0 y 1 entendiéndose el coeficiente 0 como nula confiabilidad y 1 como confiabilidad total. De acuerdo a los resultados obtenidos, para el instrumento la "r" obtenida fue de 0,87 considerándose dentro del rango de altamente confiable.

Por otra parte, para analizar los datos, se asumió la estadística inferencial como el procedimiento que facilita el análisis e 
interpretación de los resultados obtenidos a través de la fase de recolección; el análisis de los datos, se hizo a través de la estadística descriptiva, los mismos fueron procesados a partir de un baremo de interpretación, este se construyó sobre la base de las puntuaciones utilizadas en la escala, la cual va de 1 a 5 .

\section{Resultados y discusión}

\subsection{Identificación}

Luego de la codificación y tabulación de los registros, se presentan los resultados. En cuanto al elemento identificación, de la variable compromiso organizacional, se puede apreciar en la Tabla 1, que el 93\% de los sujetos encuestados definieron como muy presentes a los componentes que forman parte de este elemento, tales como las metas, los objetivos, los valores y las políticas. El resto de los resultados se concentró en la categoría presente con un $7 \%$. La media aritmética de 64,17 se situó en la categoría de mayor concentración porcentual. La desviación estándar de 5,31 define una moderada dispersión de los puntajes.

Tabla 1

Identificacion organizacional

\begin{tabular}{lllll}
\hline Categorías & Rangos & Frecuencia & Proporción & $\mathbf{\%}$ \\
\hline Muy presente & $55>-\leq 75$ & 144 & 0,93 & 93 \\
Presente & $35>-\leq 55$ & 11 & 0,07 & 7 \\
Poco presente & $15>-\leq 35$ & 0 & 0 & 0 \\
Totales & Sumatoria & Promedio & Desv. Std. & Mediana \\
& $\mathbf{9 . 9 4 7}$ & $\mathbf{6 4 , 1 7}$ & $\mathbf{5 , 3 1}$ & $\mathbf{6 4}$ \\
\hline
\end{tabular}

Fuente: Elaboración propia, 2020.

En primer lugar, se analizó la dimensión identificación organizacional, para la cual se estableció como primer indicador a las metas; al respecto, las unidades en estudio estuvieron de acuerdo en un $81 \%$ en afirmar que este elemento está muy presente en el proceso de identificación. Según Newstrom (2011), las metas son todas aquellas formulaciones completas de logros que la organización propone alcanzar en determinados periodos.

Siguiendo con los indicadores de la dimensión identificación, se habla de los objetivos, que se categorizaron en un $80 \%$ como muy presentes dentro de ésta. Corroborando el comportamiento del indicador, afirman Robbins y Coulter (2014) que los objetivos, son convicciones básicas de que un modo especifico de conducta o estado final de existencia, es personal o socialmente preferible a otro.
Muy relacionado con el aspecto precedente, está el tema de los valores. En relación a ellos, los sujetos encuestados afirmaron que los mismos están muy presentes $(89 \%)$ en el proceso de identificación, coincidiendo con García, et al. (2017b) cuando plantea que los valores: Son características particulares de una sociedad, formada por un conjunto de normas que están íntimamente cohesionadas con la cultura de los individuos y su manera de relacionarse con otros y que eso es transferible para cualquier ámbito donde éste se desarrolle.

Para completar el análisis de estos factores, los encuestados refirieron en una proporción del $79 \%$, que se alineaban con las políticas de la organización, que estos factores estaban muy presentes en su identificación con la misma. Sintetizando las ideas acerca de la dimensión identificación organizacional, 
considerada como la primera fase del compromiso, se observó en las organizaciones estudiadas que los elementos que la componen, a juicio del 93\% (ver Tabla 1) de sus unidades en estudio, están muy presentes.

\subsection{Involucramiento}

Los datos señalados en la Tabla 2, se refieren a los resultados del involucramiento en el trabajo de la variable compromiso organizacional, ésta muestra que el $99 \%$ de los encuestados definieron como muy presente esta fase de la variable con respecto a los empleados de las franquicias de comida rápida en estudio y tan solo un $1 \%$ en la categoría presente; los cuales son definidos por las decisiones, la participación, los esfuerzos, el rendimiento y el interés. El promedio de 74,20 se sitúa casi en el medio de la amplitud de rango de la categoría de mayor observación frecuencial (muy presente). La desviación estándar de 14,48 define una gran dispersión de los datos; lo que sugiere la inestabilidad de la media aritmética como media de tendencia central.

Tabla 2

Involucramiento

\begin{tabular}{ccccc}
\hline Categorías & Rangos & Frecuencia & Proporción & \% \\
\hline Muy presente & $62>-\leq 85$ & 154 & 0,99 & 99 \\
Presente & $40>-\leq 62$ & 1 & 0,01 & 1 \\
Poco presente & $17>-\leq 40$ & 0 & 0 & 0 \\
Totales & Sumatoria & Promedio & Desv. Std. & Mediana \\
& $\mathbf{1 1 . 5 0 1}$ & $\mathbf{7 4 , 2 0}$ & $\mathbf{1 4 , 4 8}$ & $\mathbf{7 3}$ \\
\hline
\end{tabular}

Fuente: Elaboración propia, 2020.

Según Robbins y Coulter (2014), este aspecto requiere de la participación activa del empleado en lo referente a la toma de decisiones importantes dentro de la organización, mediante su sentido de obligación y lealtad, traduciéndose en un mayor esfuerzo dirigido al beneficio de la misma. Al respecto, se examinó en primer término el indicador decisiones, para el cual el $82 \%$ precisó estar muy presente en este contexto. Similar comportamiento presenta el indicador participación, cuando el 91\% de los encuestados afirmó muy presente este aspecto.

En lo referido al indicador esfuerzos, el 91\% de los encuestados manifestó que está muy presente en la fase de involucramiento. Newstrom (2011), opina que este deseo se manifiesta en el impulso y el esfuerzo de los empleados hacia la eficacia personal; con base a la certeza de poseer las capacidades necesarias para desempeñar una tarea, cumplir las expectativas puestas en determinadas funciones, realizar una contribución significativa o enfrentar exitosamente una situación difícil, los empleados llevan a cabo cualquier esfuerzo personal para sacar adelante a sus organizaciones y llevarlas hacia el éxito.

Lo anterior conlleva al resultado del indicador interés, para el cual el $92 \%$ de las unidades en estudio manifestaron estar muy presente en el involucramiento con el trabajo; en cuanto al rendimiento hacia la organización; las unidades estudiadas manifestaron que, en un $78 \%$ el rendimiento está muy presente en estas organizaciones.

Al respecto, según Murrel y Meredith (2002), el rendimiento se concibe como el proceso mediante el cual las personas cumplen con las pautas de conductas exigidas por la organización, tocando aspectos primordiales como las metas organizacionales, su visión y cultura. En resumen, los elementos que constituyen el involucramiento están muy presentes en un $99 \%$ (ver Tabla 2), tal y como 
lo destacan las unidades de estudio en el contexto de investigación seleccionado.

\subsection{Sentido de pertenencia}

De acuerdo a lo expresado en la Tabla 3 , se expresa el sentido de pertenencia, concebida como lealtad hacia la organización, incluyendo items concernientes como la puntualidad, la honestidad, la justicia y la moral. Al reconocer los resultados obtenidos, se divisó una mayoría del 97\%, quienes respondieron como muy presente a esta dimensión como fase de la variable en estudio dentro de las empresas seleccionadas. Con respecto al 3\% restante, este se ubicó dentro de la categoría presente. El promedio aritmético de 60,23 cercano a los valores centrales de la categoría con mayor índice porcentual. La desviación entandar de 3,96 señala una moderada dispersión entre las categorías.

\section{Tabla 3}

Sentido de pertenencia

\begin{tabular}{ccccc}
\hline Categorías & Rangos & Frecuencia & Proporción & \% \\
\hline Muy presente & $51>-\leq 70$ & 150 & 0,97 & 97 \\
Presente & $33>-\leq 51$ & 5 & 0,03 & 3 \\
Poco presente & $14>-\leq 33$ & 0 & 0 & 0 \\
Totales & Sumatoria & Promedio & Desv. Std. & Mediana \\
\hline
\end{tabular}

Fuente: Elaboración propia, 2020.

Según los autores analizados en el estudio, este aspecto se concibe a través de la presencia de indicadores como puntualidad, honestidad, justicia y moral, destacados en la presente. Con respecto a la puntualidad, los encuestados, en un $92 \%$, manifestaron estar muy presente en las organizaciones en estudio, asimismo, la situación se presentó para el indicador honestidad, muy presente para el $84 \%$ de los interesados, justicia, con alta presencia para el $94 \%$ y moral, con similar comportamiento para el $89 \%$ de las unidades en estudio.

A este respecto, Robbins y Coulter (2014) conciben la puntualidad como todo aquello que se lleva en forma metódica, diligente y asidua, teniéndose como norte el mejor desenvolvimiento de la organización. Newstrom (2011), indica que la honestidad, involucra una serie de calificativos como la honradez, la integridad, el decoro, moralidad, condiciones estas para que el compromiso se dé.
Por estas razones, los aspectos que tienen que ver con la aprobación y asunción de la filosofía organizacional y el cumplimiento de normas, son cónsonos con la fase de sentido de pertenencia, situación que se presenta en buena proporción en las organizaciones investigadas, cuando el 97\% (ver Tabla 3) considera estos elementos muy presentes.

\subsection{Factores demográficos}

En la Tabla 4, se muestran el ordenamiento descriptivo de los datos del indicador demográfico. Al respecto, el $86 \%$ de los sujetos encuestados categorizaron dicho factor como muy presente en los factores del ausentismo laboral de las organizaciones en estudio. La media aritmética de 17,79 se localiza en la categoría de mayor registro porcentual. La desviación estándar de 1,64 señala una pequeña dispersión de los datos entre las categorías Muy Presente y Presente. 
Tabla 4

Factores demografico del ausentismo laboral

\begin{tabular}{ccccc}
\hline Categorías & Rangos & Frecuencia & Proporción & $\%$ \\
\hline Muy presente & $15>-\leq 20$ & 134 & 0,86 & 86 \\
Presente & $9>-\leq 15$ & 21 & 0,14 & 14 \\
Poco presente & $4>-\leq 9$ & 0 & 0 & 0 \\
Totales & Sumatoria & Promedio & Desv. Std. & Mediana \\
& $\mathbf{2 . 6 9 6}$ & $\mathbf{1 7 , 7 9}$ & $\mathbf{1 , 6 4}$ & $\mathbf{1 8}$ \\
\hline
\end{tabular}

Fuente: Elaboración propia, 2020.

Bajo este contexto, se tiene que los encuestados, en una proporción significativa $(86 \%)$ indicaron que estos factores están muy presentes en dichas organizaciones como causa de ausentismo laboral, en consonancia con López, et al. (2005), quien señala que las variables sociodemográficas constituyen un porcentaje importante del ausentismo. El autor analiza aspectos como el sexo, la edad, el estado civil, la existencia de hijos, la antigüedad y la profesión, destacando los tres primeros como potenciadores del ausentismo.

Siguiendo con este indicador, con respecto a la edad, hay una clara relación entre este factor y el ausentismo. Indican Mesa y Kaempffer (2004); así como Peiró, et al. (2008), que es mayor cuando los trabajadores son jóvenes. La gente joven usualmente tiene poca experiencia laboral que las personas mayores; en tanto que, la gente que tiene más experiencia ha desarrollado a lo largo de su vida mejores estrategias de afrontamiento hacia los estímulos estresantes y sus expectativas profesionales son más reales.

\subsection{Distancia al trabajo}

Para agotar la discusión con respecto a los indicadores de la dimensión factores de ausentismo laboral, aparece el último indicador estudiado, denominado distancia al trabajo. A este efecto, los resultados permiten afirmar que el $88 \%$ de los encuestados lo señalan como un aspecto fuertemente presente en las organizaciones de comida rápida. $\mathrm{Al}$ respecto, Amorós (2007), afirma que la relación entre las faltas al trabajo y la distancia a él parece obvia, sobre todo en una ciudad tan complicada y ausente de sistemas de transporte accesibles y veloces como es Barranquilla.

Tabla 5

Distancia del trabajo

\begin{tabular}{lllll}
\hline Categorías & Rangos & Frecuencia & Proporción & $\%$ \\
\hline Muy presente & $11>-\leq 15$ & 135 & 0.88 & 88 \\
Presente & $7>-\leq 11$ & 20 & 0.12 & 12 \\
Poco presente & $3>-\leq 7$ & 0 & 0 & 0 \\
Totales & Sumatoria & Promedio & Desv. Std. & Mediana \\
& $\mathbf{1 9 9 3}$ & $\mathbf{1 3 . 0 7}$ & $\mathbf{1 . 3}$ & $\mathbf{1 3}$ \\
\hline
\end{tabular}

Fuente: Elaboración propia, 2020. 
Tal y como lo describe Amorós (2007), existe una relación muy clara entre aquellas empresas que promueven como política de reclutamiento y para disminuir el ausentismo, la contratación de su personal en las cercanías de su localidad, en el caso de que esto pueda manejarse como una alternativa y la mano de obra se encuentre a la mano para lograr este cometido. Para ello, se destaca la relación entre las variables, a través del método de Spearman dado que ambas variables se han medido a través de una escala estadística ordinal (ver Tabla 6).

Tabla 6

Cálculo de rangos de Spearman de ambas variables

\begin{tabular}{cccc}
$\sum$ Rgs X & $\sum$ Rgs Y & $\sum \neq$ Rgs & $\sum(\mathbf{X}-\mathbf{Y}) 2$ \\
\hline 30.783 & 14.160 & 0 & $72.126,50$ \\
\hline
\end{tabular}

Fuente: Elaboración propia, 2020.

El procedimiento fue ejecutado en el programa SPSS, versión XXII, donde el resultado del cálculo de coeficiente de correlación Spearman fue de 0,884 , lo que indica teórica y estadísticamente cuatro (04) condiciones: Primero, existe relación entre ambas variables; segundo, que su dirección es directamente proporcional entre ambas, es decir, en el mismo sentido; tercero, la magnitud o intensidad es considerable y cuarto, que en la determinación de dicho coeficiente de correlación; el 78,14 por ciento de las varianzas de la variable Compromiso organizacional se tuvieron en cuenta para verificar la conducta de la variable ausentismo laboral.

\section{Conclusiones}

El estudio orientado a analizar los elementos del compromiso organizacional y su relación con los factores sociológicos del ausentismo laboral, permite corroborar que los elementos del compromiso están muy presentes en el proceso de integración laboral de las franquicias de comida rápida en Barranquilla, Colombia, generando un proceso de identificación organizacional, involucramiento en el trabajo y la lealtad, indicando que existe un gran sentido de pertenencia.

Esto indica, que el compromiso se asocia a características particulares de una sociedad, formada por un conjunto de normas que están íntimamente cohesionadas con la cultura de los individuos y su manera de relacionarse con otros y que eso es transferible para cualquier ámbito donde éste se desarrolle, tal como se visualiza en las franquicias objeto de estudio. Desde estas perspectivas, los elementos del compromiso organizacional no trabajan de forma independiente o aislada, sino entrelazada, interactuando entre sí, a través de la suma de factores individuales y organizacionales; estos factores o variables intervinientes, ayudan a los empleados a esmerarse en su trabajo, buscando la calidad de todo lo concerniente a su labor.

Con respecto a los factores demográficos que condicionan el ausentismo, se estima, que cada individuo tiene una estructura mentalmente diferente, actuando de acuerdo a sus capacidades sobre las fuentes de emociones interna y externas, y a los procesos bio-psico-sociales, donde algunos tienen la capacidad de responder satisfactoriamente ante las necesidades que surgen desde su vida diaria. Es así como se concluye, que dentro de los factores se localizan la edad, el género, el estado civil, cada uno de ellos o en conjunto pueden afectar el nivel de estrés manejable, lo que en caso de no poder hacerlo es probable 
que desencadene en problemas de salud y, por ende, sea el motivo de ausentismo en el lugar de trabajo.

Se estima, que las variables sociodemográficas constituyen un porcentaje importante del ausentismo, dado que se evidencia que el género, la edad, el estado civil, la existencia de hijos, los horarios de trabajo y la alta presión en aras de la atención al cliente, son potenciadores del ausentismo laboral. Además de la ubicación geográfica de la vivienda del trabajador, o distancia del sitio del trabajo. Dado que, la mayoría de los empleados, viven en zonas rurales y ubicación en la zona sur del Distrito de Barranquilla y se han empleado en la zona Norte de la ciudad.

Consecuentemente, se pudo determinar la existencia de una correlación entre el compromiso organizacional y el ausentismo laboral; y en los empleados de las cadenas de comida rápida, se obtuvo como resultado una relación que indica que existe compromiso organizacional por parte de los mismos, y que su ausencia al trabajo está referida solo a situaciones muy puntuales, que surgen como elementos demográficos.

\section{Referencias bibliográficas}

Amorós, E. (2007). Comportamiento organizacional: En busca del desarrollo de ventajas competitivas. Universidad Católica Santo Toribio de Mogrovejo.

Cabrera, A., y Díaz, E. (2019). Influencia de las capacidades dinámicas en la cultura de alto desempeño y competitividad. SUMMA. Revista Disciplinaria en Ciencias Económicas y Sociales, 1(1), 53-67.

Durán, S. (2017). Organizaciones exitosas: Procesos multiculturales. ECOE Ediciones.

Durán, S. E., Crissien, J. E., Virviesca, J., y García, J. E. (2017). Estrategias gerencialesparalaformación deequipos de trabajo en empresas constructoras del Caribe Colombiano. Revista Espacios, 38(13), 26-28.

Durán, S-E., García-Guiliany, J-E., y ParraFernández, M. (2018). Diversidad cultural y fomento de valores en la convivencia ciudadana. Revista Búsqueda, 4(19), 102-110.

García, J., Durán, S. E., y Prieto, R. (2017). Políticas de gestión de talento humano para el desarrollo de competencias gerenciales en empresas metalmecánica. FACE: Revista de la Facultad de Ciencias Económicas y Empresariales, 17(2), 130-141.

García, J. E., Durán, S. E., Parra, M. A., y Márceles, V. S. (2017a). Dirección estratégica del talento humano para el fomento de valores en los cuerpos policiales venezolanos. Revista Espacios, 38(32), 16-28.

García, J. E., Durán, S. E., Cardeño, E., Prieto, R., García, E., y Paz, A. (2017b). Proceso de planificación estratégica: Etapas ejecutadas en pequeñas $\mathrm{y}$ medianas empresas para optimizar la competitividad. Revista Espacios, 38(52), 1-14.

Herriegel, D., y Slocum, J. W. (2010). Comportamiento organizacional. Editorial Thompson.

López, Y. J., García, J., y Cardeño, E. (2017). Dirección estratégica: aplicación en empresas filiales de la corporación eléctrica nacional de Venezuela. Revista Espacios, 38(59), 16-28.

López, M., Rodríguez, A., Fernández, M., Marcos, S., Martinón, F., y Martinón, J. M. (2005). Síndrome de desgaste profesional en el personal asistencial pediátrico. Anales de Pediatría, 62(3), 248-251. https://doi. org/10.1157/13071839

Martínez, J. (2016). Factores externos 
determinantes de las decisiones de inversión a largo plazo en el sector farmacéutico. TELOS. Revista de Estudios Interdisciplinarios en Ciencias Sociales, 18(3), 415-430.

Martínez-Ventura, J., Cardeño-Portela, E., Ramírez-Cardeño, W., y Durán, S. (2017). Liderazgo transformacional como estrategia de adaptación en la gestión logística empresarial. Desarrollo Gerencial, 9(2), 140157. $\quad$ https://doi.org/10.17081/ dege.9.2.2980

Méndez, S., Morua, J., y Hernández, R. (2018). Complejidad y dinámica, la necesidad de considerarlas en la evaluación organizacional. Revista de Ciencias Sociales (Ve), XXIV(2), 9-23.

Mesa, F. R., y Kaempffer, A. M. (2004). 30 años de estudio sobre ausentismo laboral en Chile: Una perspectiva por tipos de empresas. Revista Médica de Chile, 132(9), 1100-1108. http://dx.doi.org/10.4067/S003498872004000900012

Molinera, J. F. (2006). Absentismo laboral: Causas, control y análisis, nuevas formas, técnicas para su reducción. FC Editorial. Fundación Confemetal

Murrell, K. L., y Meredith, M. (2002). Empowerment para su equipo. McGraw-Hill Interamericana.

Newstrom, J. W. (2011). Comportamiento humano en el trabajo. McGraw-Hill Interamericana.

Parra, M., Durán, S. E., Márceles, V., Yarzagaray, J., Valenzuela, I., Payares, K., Alvarino, C., Navarro, N., Charris,
P., y Ramírez, J. (2018). Factores del clima organizacional existentes en el área administrativa en empresas de Barranquilla. Revista Espacios, 39(50), 21-35.

Parra, M., Visbal, O., Durán, S. E., y Badde, G. (2019). Calidad de la comunicación y actitud de los empleados ante procesos de cambio organizacional Interdisciplinaria. Interdisciplinaria: Revista de Psicología y Ciencias Afines, 38(1), 155-170. https://doi. org/10.16888/interd.2019.36.1.11

Paz, A., Núñez, M. G., García, J., y Salom, J. (2016). Rol del liderazgo ético en organizaciones académicas. Revista Opción, 32(E-12), 148-168.

Peiró, J. M., Rodríguez-Molina, I. y GonzálezMorales, M. G. (2008). El absentismo laboral: Antecedentes, consecuencias $y$ estrategias de mejora. Universitat de València

Prieto, R., Burgos, C., García, J., y Rincón, Y. (2016). Mercadeo interno para optimizar la calidad de servicio en la banca universal. Revista Venezolana de Gerencia, 21(73), 102-119.

Robbins, S. y Coulter, M. (2014). Administración. Editorial Pearson Educación.

Rodríguez, A. (1999). Ausentismo laboral. Salud y seguridad. Boletín electrónico del Servicio de prevención de riesgos laborales, II(2).

Schemehom, J., Hunt, J., y Osborn, R. N. (2008). Comportamiento organizacional. Limusa Wiley. 